\title{
ACSL4 suppresses glioma cells proliferation via activating ferroptosis
}

\author{
JING CHENG $^{1 *}$, YAN-QIN FAN ${ }^{2 *}$, BAO-HUI LIU $^{1}$, HAN ZHOU $^{3}$, JUN-MIN WANG ${ }^{1}$ and QIAN-XUE CHEN ${ }^{1}$ \\ Departments of ${ }^{1}$ Neurosurgery and ${ }^{2}$ Nephrology, Renmin Hospital of Wuhan University, Wuhan, Hubei 430060; \\ ${ }^{3}$ Department of Neurosurgery, The Affiliated Hospital of Qingdao University, Qingdao, Shandong 266003, P.R. China
}

Received June 1, 2019; Accepted October 30, 2019

DOI: $10.3892 /$ or.2019.7419

\begin{abstract}
Acyl-CoA synthetase long-chain family member 4 (ACSL4) is a member of the long chain family of acyl-CoA synthetase proteins, which have recently been shown to serve an important role in ferroptosis. Previous studies have suggested that ferroptosis is involved in the occurrence of glioma; however, the role of ACSL4 in glioma remains unknown. In the present study, a reduction of ferroptosis in human glioma tissues and glioma cells was observed. Subsequently, it was demonstrated that the expression of ACSL4 was also downregulated in human glioma tissues and cells. A ferroptosis inhibitor and inducer were used to investigate the effects of ferroptosis on viability. The results showed that promoting ferroptosis inhibited the proliferation of glioma cells, and that the use of inducers had the reverse effect. Therefore, it was hypothesized that the reduction in ACSL4 expression may have been involved in ferroptosis and proliferation in glioma. Overexpression of ACSL4 decreased expression of glutathione peroxidase 4 and increased the levels of ferroptotic markers, including 5-hydroxyeicosatetraenoic (HETE), 12-HETE and 15-HETE. Additionally, ACSL4 overexpression resulted in an increase in lactate dehydrogenase release and a reduction in cell viability. The opposite results were observed when ACSL4 was silenced. These findings suggest that ACSL4 regulates ferroptosis and proliferation of glioma cells. To further investigate the mechanism underlying ACSL4-mediated regulation of proliferation in glioma cells, cells were treated with small interfering (si)-ACSL4 and sorafenib, a ferroptosis inducer. sorafenib attenuated the ability of siRNA-mediated silencing of ACSL4, thus improving cell viability. These results
\end{abstract}

Correspondence to: Professor Qian-Xue Chen or Professor Jun-Min Wang, Department of Neurosurgery, Renmin Hospital of Wuhan University, 99 Zhang Zhidong Road, Wuhan, Hubei 430060, P.R. China

E-mail: chenqx666@whu.edu.cn

E-mail: drwangjunmin@126.com

${ }^{*}$ Contributed equally

Key words: acyl-CoA synthetase long-chain family member 4, glioma, ferroptosis, glutathione peroxidase 4, proliferation demonstrate that ACSL4 protects glioma cells and exerts anti-proliferative effects by activating a ferroptosis pathway and highlight the pivotal role of ferroptosis regulation by ACSL4 in its protective effects on glioma. Therefore, ACSL4 may serve as a novel therapeutic target for the treatment of glioma.

\section{Introduction}

Glioma is the most common primary intracranial tumor, accounting for 50-60\% of primary tumors in the central nervous system (1). In recent years, despite improvements in treatments, the majority of patients with malignant glioma have an unfavorable outcome within 2 years of diagnosis (2). Glioblastoma is the most common and most malignant type of glioma, with the worst prognosis (3). A population-based study found that the median overall survival time of patients with glioblastoma patients was 8-14 months in the USA between 2010 and 2012 (4). Glioblastoma is highly resistant to multiple combined therapies (5), and the average survival time of patients with glioblastoma is short. According to the Chinese Glioma Genome Atlas, the median survival time of patients with glioblastoma is only 14.4 months $(6,7)$. Therefore, finding effective therapeutic strategies for patients with glioma is an urgent problem that needs to be addressed.

Ferroptosis is an iron-dependent, atypical form of cell death characterized by the accumulation of lipid peroxidation products and lethal reactive oxygen species (ROS) $(8,9)$. It is a relatively newly understood mechanism of programmed cell death discovered by the Stockwell Laboratory of the Department of Biological Sciences at Columbia University in 2012 (10). Ferroptosis has specific energy and morphological characteristics, including reduced nicotinamide adenine dinucleotide phosphate levels, normal levels of adenosine triphosphate, damaged membrane integrity, normal nucleus and atrophic mitochondria (11-13). However, relatively few studies have investigated the association between ferroptosis and glioma. Fan et al (14) demonstrated that ferroptosis inhibition accelerates proliferation and metastasis of gliomas (14). Chen et al (15) discovered that ferroptosis suppression could promote malignant transformation, proliferation and angiogenesis of gliomas (15). Therefore, the present study hypothesized that the occurrence of glioma may be associated with the reduction of ferroptosis. 
Acyl-CoA synthetase long-chain family member 4 (ACSL4) is a key factor involved in metabolic-associated diseases (16). Previous studies have found that metabolic disorders of amino acid synthesis, lipid synthesis and iron-transport result in cell death (17-19). In addition, previous studies have shown that metabolic disorders of amino acid synthesis, lipid synthesis and iron transport are involved in ferroptosis (11,20-22). ACSL4, cysteine-glutamate antiporter system and glutathione peroxidase $4(\mathrm{GPx} 4)$ are the three primary components that regulate ferroptosis (21). Recent studies have shown that ACSL 4 promotes the formation of phytosterol esters esterified from arachidonic acid (AA) and adrenaline, which is a process associated with ferroptosis $(13,23)$. ACSL4 is considered a vital regulator of ferroptosis, and overexpression of ACSL4 promotes ferroptosis (23). Therefore, it was hypothesized that a decrease in ferroptosis in glioma may be the result of reduced ACSL4 expression, and ACSL4 may be involved in the pathogenesis of glioma

The present study demonstrated the effects of ferroptosis on proliferation of glioma cells, and investigated a novel mechanism involving ACSL4. ACSL4 expression effected the proliferation of glioma cells by regulating ferroptosis. Therefore, ACSL4 may be a novel therapeutic target for the treatment of glioma.

\section{Materials and methods}

Human glioma tissues and normal human brain tissues. Brain specimens from male patients aged 43-62 years were used in the present study. Table I summarizes the clinical characteristics of the 6 patients with glioma included in the present study. The donors' brain samples were obtained from the Chinese Brain Bank Center (CBBC) at the South-Central University for Nationalities (SCUN) and exhibited no signs of clinical or post-mortem neurological disease. The brain tissue samples were collected in June 2018. The human donation plan implemented by the Wuhan Red Cross Society passed the autopsy. Consent was obtained for brain autopsy and use of the brain material according to the protocol of CBBC and the human body donation program, and medical records for research purposes were provided by the donors themselves or their relatives and approved by the Biomedical Research Ethics Committee of SCUN (approval no. 2017-SCUEC-MEC-004) Human glioma tissues were obtained at the time of surgery $(n=6)$ at the Department of Neurosurgery, Renmin Hospital of Wuhan University (Wuhan, China). The samples were collected between September 2018 and November 2018. Pathological diagnosis was confirmed independently by three neuropathologists. Procurement of tissues for the present study was approved by the Institutional Ethics Committee of the Faculty of Medicine at Renmin Hospital of Wuhan University (approval no. 2018K-C017) and written informed consent was obtained from each patient before they succumbed to the disease. In the present study, glioma tissue samples were collected from 6 glioma patients as the glioma group, and healthy brain tissue samples were collected from two healthy donors as the control group. The healthy donors were both male, with an age of 38 and 49 years. Written informed consent was obtained from these individuals. Brain tissue samples from the healthy donors were from the CBBC at the SCUN in June 2018.
Cell culture. The human glial cell line HEB was purchased from Shanghai Bioleaf Biotech Co., Ltd. The human T98G glioma cell line was purchased from the American Type Culture Collection (ATCC), and the human U87 MG and U251 glioblastoma cell lines were obtained from the Cell Bank of Type Culture Collection of the Chinese Academy of Sciences. The U87 MG cells (cat. no. TCHu138) used in the present study were originally from the ATCC; therefore, they are glioblastoma cells of unknown origin. Cells were cultured in DMEM supplemented with 10\% FBS (both from Gibco: Thermo Fisher Scientific, Inc.), $100 \mu \mathrm{g} / \mathrm{ml}$ penicillin and $100 \mu \mathrm{g} / \mathrm{ml}$ streptomycin (Sigma-Aldrich; Merck KGaA), and were incubated at $37^{\circ} \mathrm{C}$ with $5 \% \mathrm{CO}_{2}$.

Western blotting. Western blot analysis was performed as described previously (24). Briefly, brain tissues (glioma tissue and healthy brain tissue) and glioma cells were lysed in ice-cold RIPA buffer (50 mM Tris- $\mathrm{HCl}$ pH 7.4, $150 \mathrm{mM}$ $\mathrm{NaCl}, 1 \%$ Triton X-100, $0.25 \%$ deoxycholate, $1.5 \mathrm{mM} \mathrm{MgCl}_{2}$, $1 \mathrm{mM}$ EGTA, $1 \mathrm{mM}$ phenylmethylsulfonyl fluoride, $10 \mathrm{mM}$ $\mathrm{ZnAF}, 10 \mathrm{mM}$ pervanadate, $10 \mu \mathrm{g} / \mathrm{ml}$ leupeptin and $10 \mu \mathrm{g} / \mathrm{ml}$ aprotinin) and incubated for $30 \mathrm{~min}$. The protein concentration was determined using the BCA method. The samples lysate was heated at $100^{\circ} \mathrm{C}$ for $10 \mathrm{~min}$ after being mixed with sample loading buffer. The $10 \mu \mathrm{g}$ protein samples were equally loaded on $10 \%$ SDS-PAGE and then transferred to PVDF membranes (EMD Millipore). Following blocking with 5\% non-fat milk in Tris-buffer at room temperature for $2 \mathrm{~h}$, the PVDF membranes were incubated with the following primary antibodies overnight at $4^{\circ} \mathrm{C}$ : ACSL4 mouse monoclonal antibody $(1: 1,000$; cat. no. sc-365230; Santa Cruz Biotechnology, Inc.), GPx4 mouse monoclonal antibody (1:1,000; cat. no. sc-166570; Santa Cruz Biotechnology, Inc.) and GAPDH rabbit monoclonal antibody (1:3,000; cat. no. ab8245; Abcam). The membranes were subsequently incubated with a horseradish peroxidase-conjugated goat anti-mouse IgG secondary antibody (1:10,000; cat. no. ab205719; Abcam) at room temperature for $1 \mathrm{~h}$ and signals were visualized using super-signal West Femto (Pierce; Thermo Fisher Scientific, Inc.). Densitometry analysis was performed using ImageJ software (version 1.46; National Institutes of Health).

Immunofluorescence assay. Fresh frozen human glioma tissue samples and healthy brain tissue samples, which were stored at $-80^{\circ} \mathrm{C}$, were fixed in $4 \%$ paraformaldehyde at $4^{\circ} \mathrm{C}$ for $24 \mathrm{~h}$ and then transferred into $30 \%$ sucrose solution in $100 \mathrm{~mol} / \mathrm{ml}$ PBS at $4^{\circ} \mathrm{C}$ for $72 \mathrm{~h}$. Subsequently, the glioma tissue samples were kept in $4 \%$ paraformaldehyde solution at $4^{\circ} \mathrm{C}$ overnight. The tissues were then cut into $16-\mu \mathrm{m}$ sections using a Leica VT1000S vibratome (Leica Microsystems GmbH). Brain sections were stained with primary mouse anti-ACSL4 (1:100; cat. no. sc-365230; Santa Cruz Biotechnology, Inc.) or mouse anti-GPx4 (1:100; cat. no. sc-166570; Santa Cruz Biotechnology, Inc.) antibodies overnight at $4^{\circ} \mathrm{C}$. Goat anti-mouse IgG1 cross-adsorbed secondary antibody, Alexa Fluor 488 (1:1,000; cat. no. A-21121; Molecular Probes; Thermo Fisher Scientific, Inc.) was incubated with the tissues at $37^{\circ} \mathrm{C}$ in the dark for $2 \mathrm{~h}$ as the secondary antibody. Tissues were counterstained with Hoechst nuclear dye (Thermo Fisher Scientific, Inc.) at room temperature for $1 \mathrm{~min}$ and sections 
Table I. Clinical characteristics of the 6 patients with glioma.

\begin{tabular}{|c|c|c|c|c|c|c|}
\hline Patient & Sex & Age, years & Histological typing & Genetic typing & Grade $^{\mathrm{a}}$ & Location \\
\hline 1 & Male & 43 & Oligodendroglioma & $\begin{array}{l}\text { IDH-mutant, } 1 \mathrm{p} / 19 \mathrm{q} \\
\text { combined deletion }\end{array}$ & II & Left temporal lobe \\
\hline 2 & Male & 47 & Diffuse astrocytoma & IDH-mutant & II & Occipital lobe \\
\hline 3 & Male & 49 & Glioblastoma & IDH-wild type & IV & Occipital lobe \\
\hline 4 & Male & 51 & Anaplastic oligodendroglioma & $\begin{array}{l}\text { IDH-mutant, } 1 \mathrm{p} / 19 \mathrm{q} \\
\text { combined deletion }\end{array}$ & III & Left temporal lobe \\
\hline 5 & Male & 50 & Glioblastoma & IDH-wild type & IV & Frontal lobe \\
\hline 6 & Male & 62 & Anaplastic oligodendroglioma & $\begin{array}{l}\text { IDH-mutant, } 1 \mathrm{p} / 19 \mathrm{q} \\
\text { combined deletion }\end{array}$ & III & Right temporal lobe \\
\hline
\end{tabular}

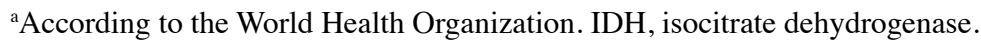

were images were captured using a fluorescence microscope (magnification, $\mathrm{x} 40$ ). Immunofluorescence analysis was performed using ImageJ software (version 1.46; National Institutes of Health).

Transient transfection. pcDNA3.1-ACSL4 plasmid (Shanghai GeneChem Co., Ltd.). negative control pcDNA3.1 vector plasmid (Shanghai GeneChem Co., Ltd.), small interfering (si)-ACSL4 (cat. no. sc-60619; Santa Cruz Biotechnology, Inc.) and negative control siRNA (cat. no. sc-37007; Santa Cruz Biotechnology, Inc.) were used for transfection. The cells were transfected with $1 \mu \mathrm{g} / \mu \mathrm{l}$ pcDNA3.1-ACSL4 plasmid, $1 \mu \mathrm{g} / \mu \mathrm{l}$ negative control pcDNA3.1 plasmid, $3 \mu \mathrm{mol} / 1$ si-ACSL4 or $3 \mu \mathrm{mol} / 1$ negative control siRNA using Lipofectamine ${ }^{\mathrm{TM}} 3000$ (Invitrogen; Thermo Fisher Scientific, Inc.) according to the manufacturer's protocol. Prior to transfection, cells were plated in 6- or 96-well plates and grown to 40-50\% confluence. Cells were transfected for $48 \mathrm{~h}$ before adding the ferroptosis inhibitor or agonist.

5-hydroxyeicosatetraenoic (HETE) assay. 5-HETE, an ACSL4-mediated product of AA oxidation in lysates of intestines and cells (25), was assessed using a 5-HETE ELISA kit (cat. no. CED739Ge; Uscn Life Sciences, Inc.), according to the manufacturer's protocol.

12/15-HETE assay. 12/15-HETE levels were assessed using 12/15-HETE ELISA kits (cat. nos. ab133034 and ab133035; Abcam), according to the manufacturer's protocols. Each 96-well plate, contained control, blank, standard and sample wells. Each sample was assayed with a minimum of two replicates. Initially, $100 \mu \mathrm{l}$ of the appropriate diluent and $50 \mu \mathrm{l}$ assay buffer was added to the non-specific binding (NSB) wells and $100 \mu \mathrm{l}$ of the appropriate diluent was added to the B0 $(0 \mathrm{pg} / \mathrm{ml}$ standard) wells. Subsequently, $50 \mu 1$ 12/15-HETE alkaline phosphatase conjugate was added to NSB, B0, standard and sample wells and $50 \mu \mathrm{l} 12 / 15$-HETE antibody was added to the B0, standard and sample wells. The plates were incubated on a plate shaker for $2 \mathrm{~h}$ at $56 \mathrm{x} \mathrm{g}$ at room temperature, after which, the contents of the wells were washed three times. Following washing, $5 \mu 1$ 12/15-HETE alkaline phosphatase conjugate was added to the total activity wells and $200 \mu 1$
pNpp substrate solution was added to each well. Plates were incubated at $3^{\circ} \mathrm{C}$ for $3 \mathrm{~h}$ without shaking. To stop the reactions, $50 \mu \mathrm{l}$ stop solution was added to each well. Absorbance was measured at $405 \mathrm{~nm}$ using a microplate reader and the mean net absorbance measurement for each well was calculated using the manufacturer-supplied formula to calculate the levels of 12/15-HETE.

Lactate dehydrogenase ( $\mathrm{LDH}$ ) assay. $\mathrm{LDH}$ is a cytoplasmic enzyme that is retained by viable cells with an intact plasma membrane and released from cells with damaged membranes (26). LDH release was measured using a colorimetric CytoTox 96 Cytotoxicity kit (Promega Corporation) according to the manufacturer's protocol. Maximum LDH release levels were measured by treating the cultures with $10 x$ lysis solution to measure complete lysine levels in the cells. Absorbance was measured at $490 \mathrm{~nm}$ using a 96-well plate reader (Molecular Devices, LLC). LDH release, as a percentage, was determined by calculating the ratio of experimental LDH release to maximal LDH release according to the manufacturer's protocol (27).

Cell Counting Kit-8 (CCK-8) cell viability assay. Cell viability was determined using a CCK-8 assay (Dojindo Molecular Technologies, Inc.) according to the manufacturer's protocol. Cells were seeded in 96-well plates at a density of 5,000/well. CCK- 8 was added at $0,24,48$ and $72 \mathrm{~h}$ and incubated at $37^{\circ} \mathrm{C}$ for $2 \mathrm{~h}$ for the measurement of absorbance. Absorbance was measured at $450 \mathrm{~nm}$ using a microplate reader (Bio-Rad Laboratories, Inc.).

Chemicals and reagents. T98G, U87 and U251 cells were treated with the ferroptosis inducers sorafenib $(5 \mu \mathrm{M})$ or erastin $(10 \mu \mathrm{M})$, or the ferroptosis inhibitors ferrostatin-1 $(1 \mu \mathrm{M})$ or U0126 $(5 \mu \mathrm{M})$, or an equivalent volume of DMSO for 3 days at $37^{\circ} \mathrm{C}$ with $5 \% \mathrm{CO}_{2}$. The cells were divided into six groups: i) Control group, untreated cells; ii) control + DMSO group, cells with equivalent volume of DMSO $(5 \mu \mathrm{M})$; iii) sorafenib group, cells treated with sorafenib $(5 \mu \mathrm{M})$; iv) siRNA negative control group, cells treated with $2 \mu \mathrm{mol} / 1$ siRNA negative control; v) si-ACSL4 group, cells with $2 \mu \mathrm{mol} / 1$ si-ACSL4; and vi) si-ACSL4 + sorafenib group, cells treated with $2 \mu \mathrm{mol} / 1$ 
si-ACSL4 and sorafenib $(5 \mu \mathrm{M})$. Erastin was purchased from Hycultec $\mathrm{GmbH}$. Sorafenib was purchased from LC Laboratories. Ferrostatin-1 and U0126 were purchased from Santa Cruz Biotechnology, Inc.

Statistical analysis. The data and statistical analysis in the present study was performed according to the recommendations on experimental design and analysis (28). Group sizes per experiment were based on a power analysis. Student's t-test or one-way ANOVA were used where appropriate to determine significance. Bonferroni's post-hoc test was used where appropriate. All results are presented as the mean \pm standard error of the mean. $\mathrm{P}<0.05$ was considered to indicate a statistically significant difference.

\section{Results}

Ferroptosis is reduced in human glioma tissues and glioma cells. It has been demonstrated that ferroptosis is involved in the pathopoiesis of glioma (29). However, research in this area remains limited. Therefore, in the present study, the levels of ferroptosis in human glioma tissues and glioma cells were measured. GPx4, 12-HETE and 15-HETE are either the lipid peroxidation products or associated with the deposition of ferritin involved in ferroptosis. These molecules are increasingly recognized as markers of ferroptosis $(11,13,24)$. Therefore, GPx4, 12-HETE and 15-HETE were selected as detection indicators for ferroptosis. The higher the level of GPX4 and the lower the level of 12/15-HETE, the less ferroptosis $(11,13,24)$. In the present study, the expression of GPx4 was significantly increased in human glioma tissues compared with brain tissues from healthy patients as determined by western blotting (Fig. 1A). Significantly increased expression of GPx4 was also observed in glioma cells in vitro (Fig. 1B). Immunofluorescence staining was used to evaluate GPx4 expression in Hoechst-positive cells in human glioma tissues. The results demonstrated that GPx4 expression was significantly upregulated in glioma cells in vivo (Fig. 1C). ELISA was used to evaluate the levels of 12-HETE and 15-HETE. Both 12-HETE and 15-HETE levels were decreased in glioma cells compared with the controls (Fig. 1D and E). These results show a higher level of GPx4 and a lower level of 12/15-HETE in human glioma tissues and glioma cells compared with the healthy human tissues and glial cell. Therefore, it was suggested that ferroptosis is reduced in glioma both in vivo and in vitro.

ACSL4 is downregulated in human glioma tissues and cells. ACSL4 was previously suggested to be a key mediator of ferroptosis (11). However, to the best of our knowledge, whether ACSL4 modulates ferroptosis in glioma has not been studied. A significant downregulation of ACSL4 was observed in human glioma tissues compared with the control (Fig. 2A). In addition, immunofluorescence staining showed that ACSL4 expression in Hoechst-positive cells in human glioma tissues was significantly downregulated in glioma cells in vivo (Fig. 2B). ACSL4 expression in glioma cells was also significantly reduced compared with in glioma cells (Fig. 2C). Therefore, it was hypothesized that the reduction in ACSL4 expression may be associated with ferroptosis disorder in glioma.
Ferroptosis-induction reduces proliferation and inhibition increases proliferation in glioma cells. To examine whether ferroptosis affects proliferation, cytotoxicity (LDH) and cell survival (CCK-8) assays were performed to evaluate cell proliferation in cells treated with ferroptosis inducers or inhibitors. T98G, U87 and U251 glioma cells were used to observe the role of ferroptosis on proliferation. It is understood that the more $\mathrm{LDH}$ release, the more severe the cell damage, which results in a decrease in cell proliferation (26). Similarly, in a CCK-8 assay, the higher the indicator, the higher the cell viability (30). Treatment with the ferroptosis inducers sorafenib and erastin resulted in a significant increase in LDH release (Fig. 3A-C) and a significant decrease in the viability of glioma cells (Fig. 3G-I). This indicates that ferroptosis-induction reduces proliferation and viability. By contrast, treatment with the ferroptosis inhibitors Ferrostatin-1 and U0126 resulted in a significant decrease in $\mathrm{LDH}$ release (Fig. 3D-F) and a significant increase in viability (Fig. 3J-L). This suggests that ferroptosis-inhibition increases proliferation and viability. This is consistent with the expected results $(31,32)$. These results suggest that ferroptosis attenuates viability in glioma cells.

ACSL4 upregulates ferroptosis and downregulates proliferation in glioma cells. ACSL4 has been proposed to serve a crucial role in ferroptosis and proliferation in other animal models. ACSL4 inhibition prior to reperfusion protects against ferroptosis and cell death in intestinal ischemia/reperfusion (33). However, whether ACSL4 modulates ferroptosis and proliferation in glioma cells remains unclear. pcDNA3.1-ACSL4 and si-ACSL4 were transfected into three different cultured glioma cell lines to elucidate the effects of ACSL4 on ferroptosis and proliferation in glioma. pcDNA3.1-ACSL4-mediated overexpression of ACSL4 significantly reduced the expression of GPx4 at the protein level (Fig. 4A, E and I) and significantly increased other markers of ferroptosis, including 5-HETE, 12-HETE, and 15-HETE (Fig. 4B-D, F-H and J-L). The lower the level of GPx4 and the higher the level of HETEs, the more ferroptosis (8). The results demonstrate that ACSL4 overexpression upregulates ferroptosis. As presented in Fig. 4M-R, ACSL4 overexpression significantly increased the release of LDH and significantly reduced the viability of glioma cells, suggesting that ACSL4 overexpression downregulated proliferation in glioma cells. siRNA-mediated knockdown of ACSL4 significantly increased GPX4 protein expression (Fig. 5A, E and I) and reduced the expression of 5-HETE, 12-HETE and 15-HETE (Fig. 5B-D, F-H and J-L). This indicated that siRNA-mediated knockdown of ACSL4 increased GPX4 expression, and reduced the markers of ferroptosis. The results demonstrated that downregulation of ACSL4 decreased ferroptosis. In addition, si-ACSL4 significantly reduced the release of LDH (Fig. 5M-O) and significantly increased the viability of glioma cells (Fig. 5P-R). These results demonstrated that downregulation of ACSL4 promoted proliferation in glioma cells. Therefore, it was concluded that ACSL4 serves a vital role in regulating ferroptosis and proliferation in glioma cells.

ACSL4 inhibition promotes proliferation of glioma cells by reducing ferroptosis. As ACSL4 was demonstrated to serve a role in regulating proliferation in glioma cells, the underlying mechanisms were then determined. si-ACSL4 and the 
A

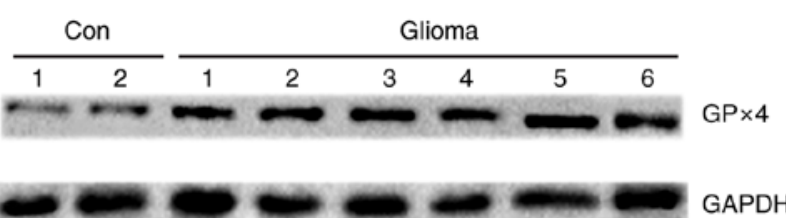

B

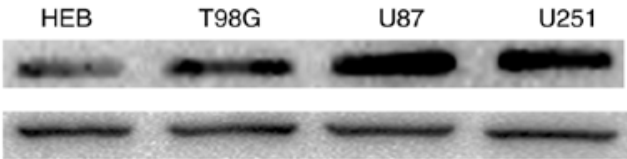

C
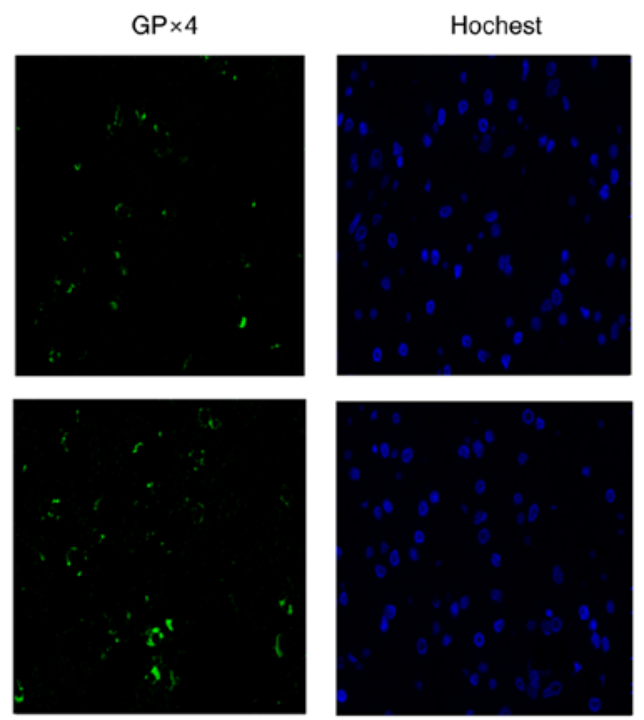

D

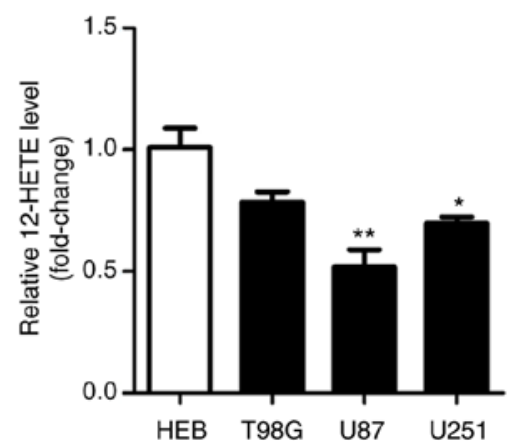

$\mathrm{GP} \times 4$

GAPDH
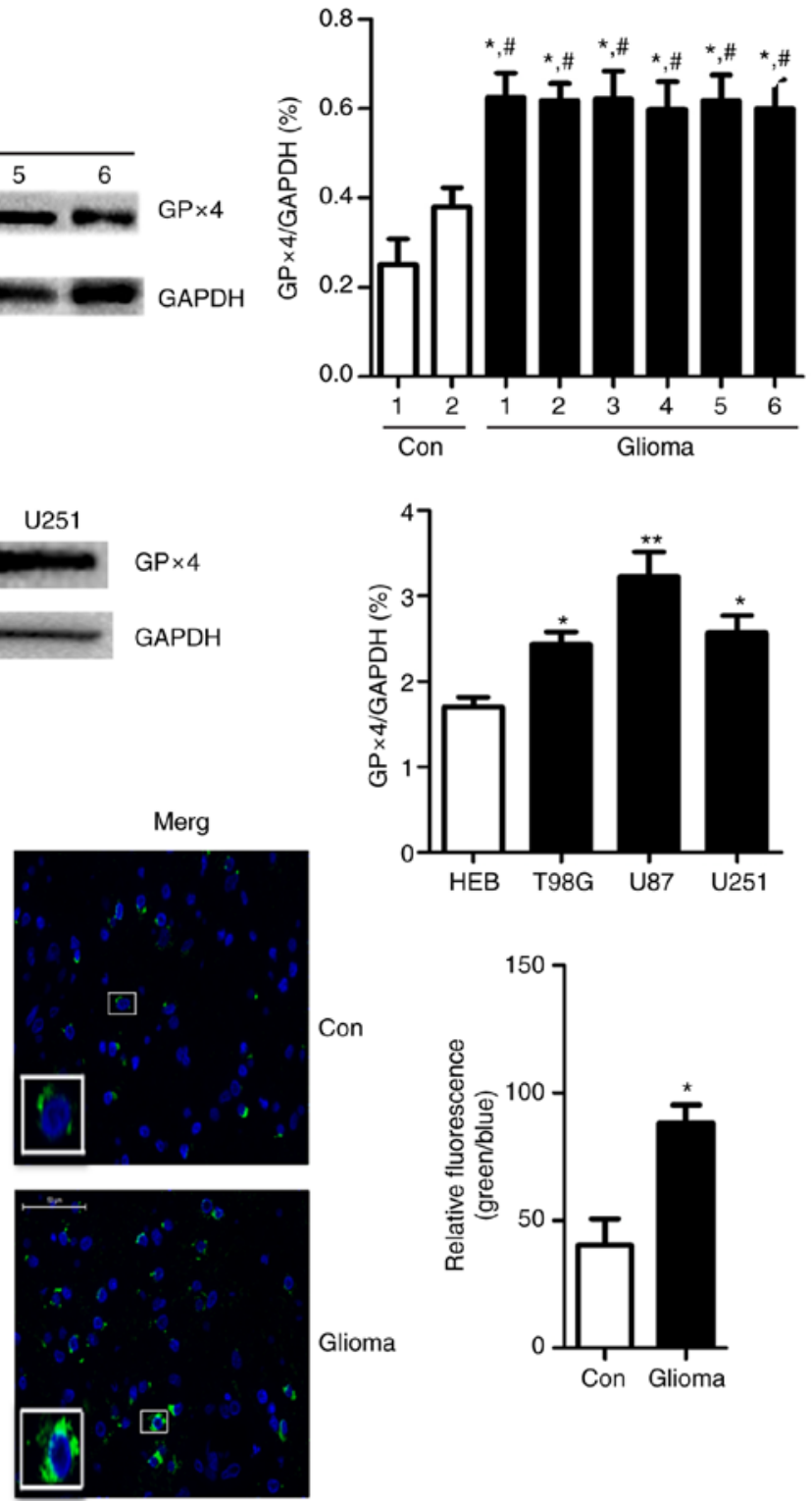

E

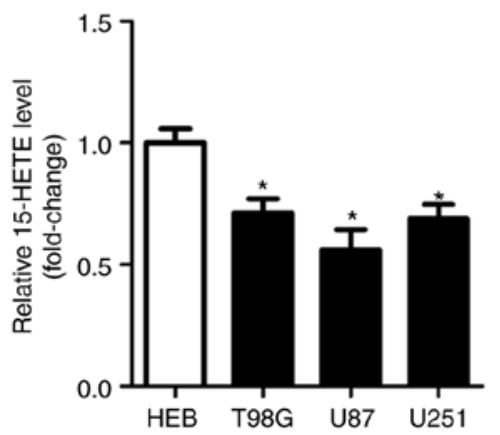

Figure 1. Expression of ferroptosis markers in human glioma tissues and cells. GPx4 expression and changes in 12-HETE and 15-HETE were detected in normal human brain tissues and glioma tissues, and in HEB glial cells and T98G, U87 and U251 glioma cells. (A) Representative western blots of GPx4 expression in normal human brain tissues and human glioma tissues, and quantification of the results. ${ }^{*} \mathrm{P}<0.05$ vs. con $1 ;{ }^{*} \mathrm{P}<0.05$ vs. con 2 . (B) Representative western blots of GPx4 expression in normal human glial cells and glioma cells, and quantification of the results. ${ }^{*} \mathrm{P}<0.05,{ }^{* *} \mathrm{P}<0.001$ vs. HEB cells. (C) Representative immunofluorescence staining of GPx4 expression in normal human brain tissues and human glioma tissues. Scale bar, $50 \mu \mathrm{m}$. Magnification of the boxed area, x 100. ${ }^{*} \mathrm{P}<0.05$ vs. con. (D) 12 -HETE levels were detected in normal human glial cells and glioma cells using a 12-HETE kit. ${ }^{*} \mathrm{P}<0.05,{ }^{* *} \mathrm{P}<0.001$ vs. HEB cells (E) 15-HETE levels were detected in normal human glial cells and glioma cells using a 15-HETE kit. ${ }^{*} \mathrm{P}<0.05$ vs. HEB cells. n=6 per group. ACSL4, acyl-CoA synthetase long-chain family member 4; GPx4, glutathione peroxidase 4; HETE, hydroxyeicosatetraenoic acid; con, control.

ferroptosis inducer Sorafenib were used to determine the mechanism by which ACSL4 regulates proliferation in glioma cells. As presented in Fig. 3, sorafenib induced ferroptosis and reduced glioma cell proliferation. It was also demonstrated that siRNA-mediated knockdown of ACSL4 could inhibit ferroptosis and promote glioma cell proliferation (Fig. 5). On the basis of 
A

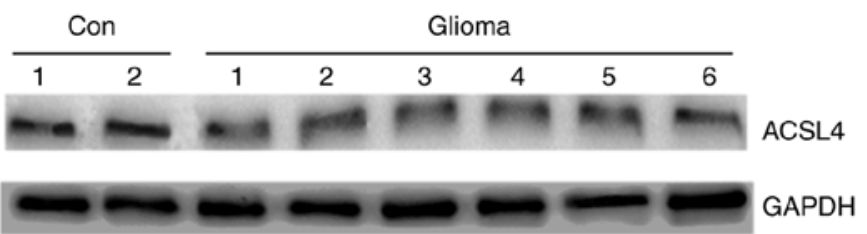

B
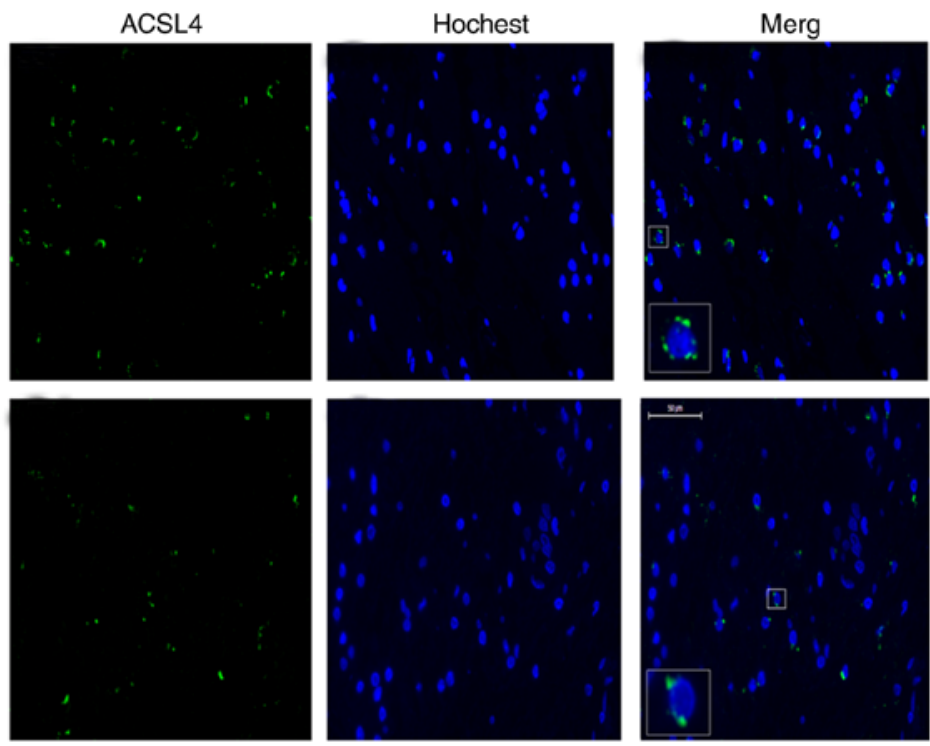

C

HEB

T98G

U87

U251

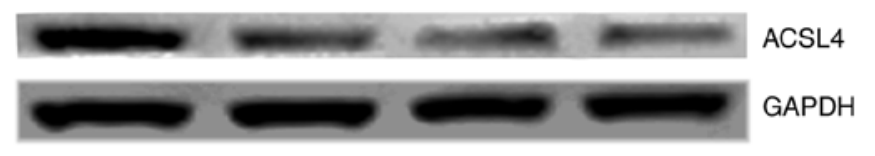

Con
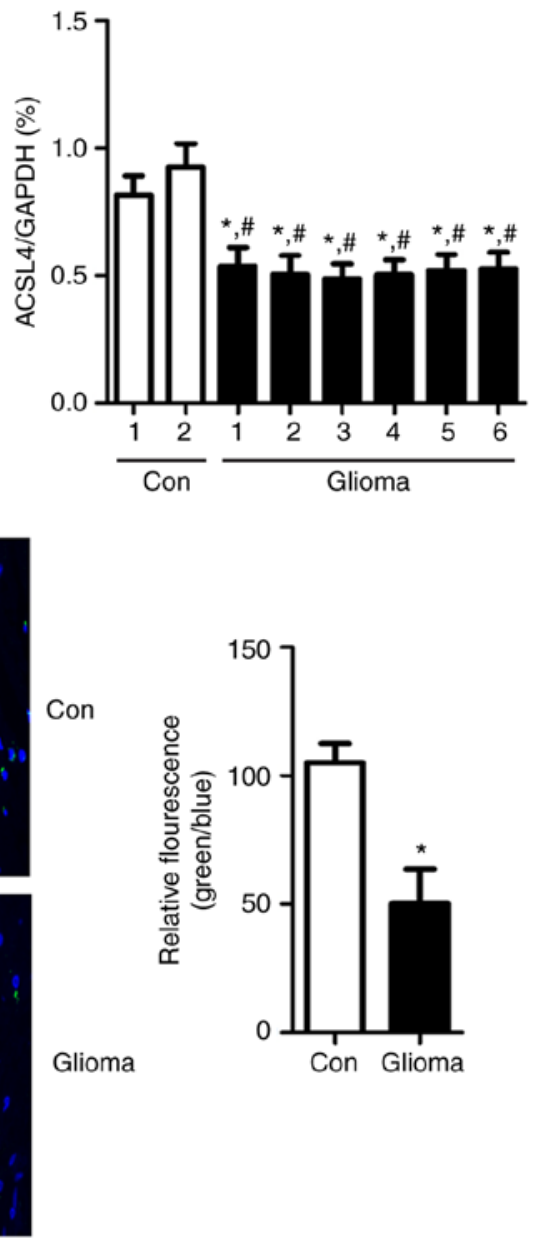

Glioma

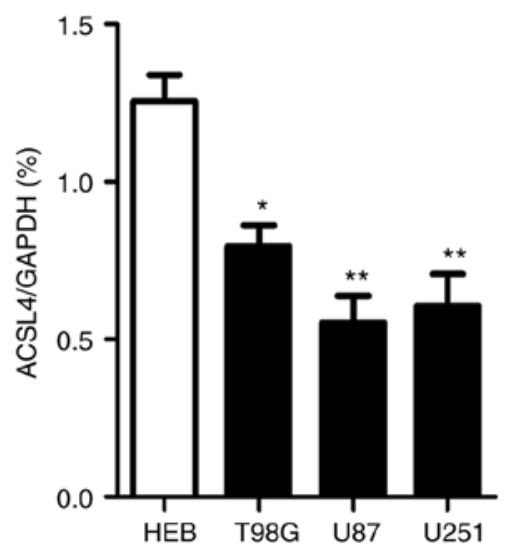

Figure 2. ACSL4 expression in human glioma tissues and glioma cells. (A) Representative western blots of ACSL4 expression in normal human brain tissues and human glioma tissues, and quantification of the results. ${ }^{*} \mathrm{P}<0.05$ vs. con $1 ;{ }^{*} \mathrm{P}<0.05$ vs. con 2 . (B) Representative immunofluorescence staining of ACSL4 expression in normal human brain and glioma tissues. Scale bar, $50 \mu \mathrm{m}$. Magnification of the boxed area, $\mathrm{x} 100 .{ }^{*} \mathrm{P}<0.05$ vs. con. (C) Representative western blots of ACSL4 expression in normal human glial and glioma cells. ${ }^{*} \mathrm{P}<0.05,{ }^{* *} \mathrm{P}<0.001$ vs. HEB cells. ACSL4, acyl-CoA synthetase long-chain family member 4; con, control.

these results, further investigation was performed. As presented in Fig. 6A-C, LDH release was significantly increased in the sorafenib group compared with the control + DMSO group and this effect could be significantly reduced by siRNA-mediated knockdown of ACSL4 in the si-ACSL4 + sorafenib group. This demonstrated that knockdown of ACSL4 could reduce sorafenib-induced ferroptosis. Furthermore, Fig. 6D-F demonstrates the viability of glioma cells in the different groups at $72 \mathrm{~h}$ after glioma cells were seeded in 96-well plates in the CCK- 8 assay. The results of the CCK- 8 assay indicated that the sorafenib-induced reduction in cell viability was reversed by siRNA-mediated knockdown of ACSL4. Taken together, these results suggest that ferroptosis is involved in the regulation of proliferation by ACSL4 in glioma cells; ACSL4 can inhibit proliferation of glioma cells by activating ferroptosis.

\section{Discussion}

Ferroptosis was first described in 2012 when Dixon et al (10) performed research on a small molecule termed erastin. A 

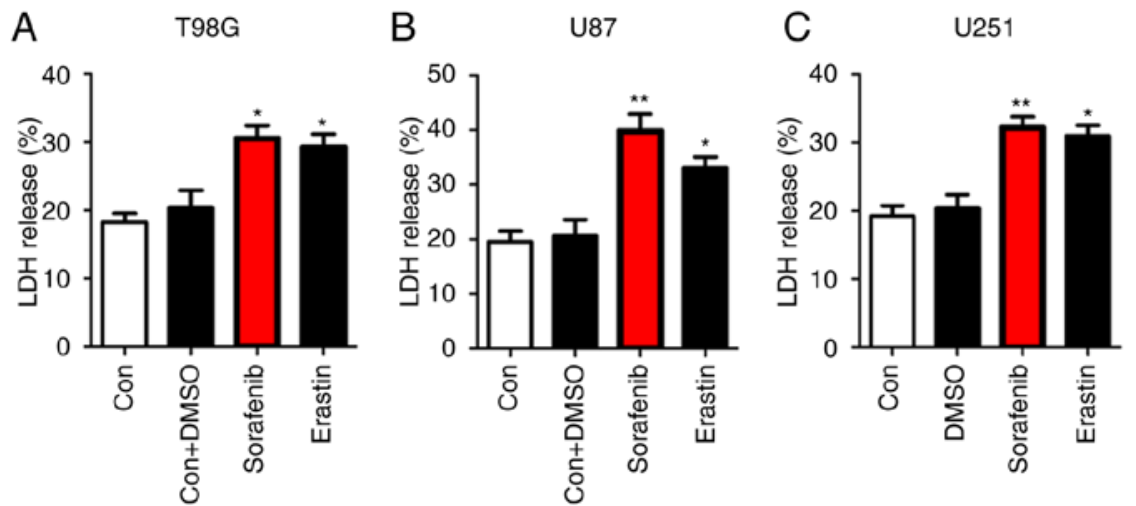

D T98G
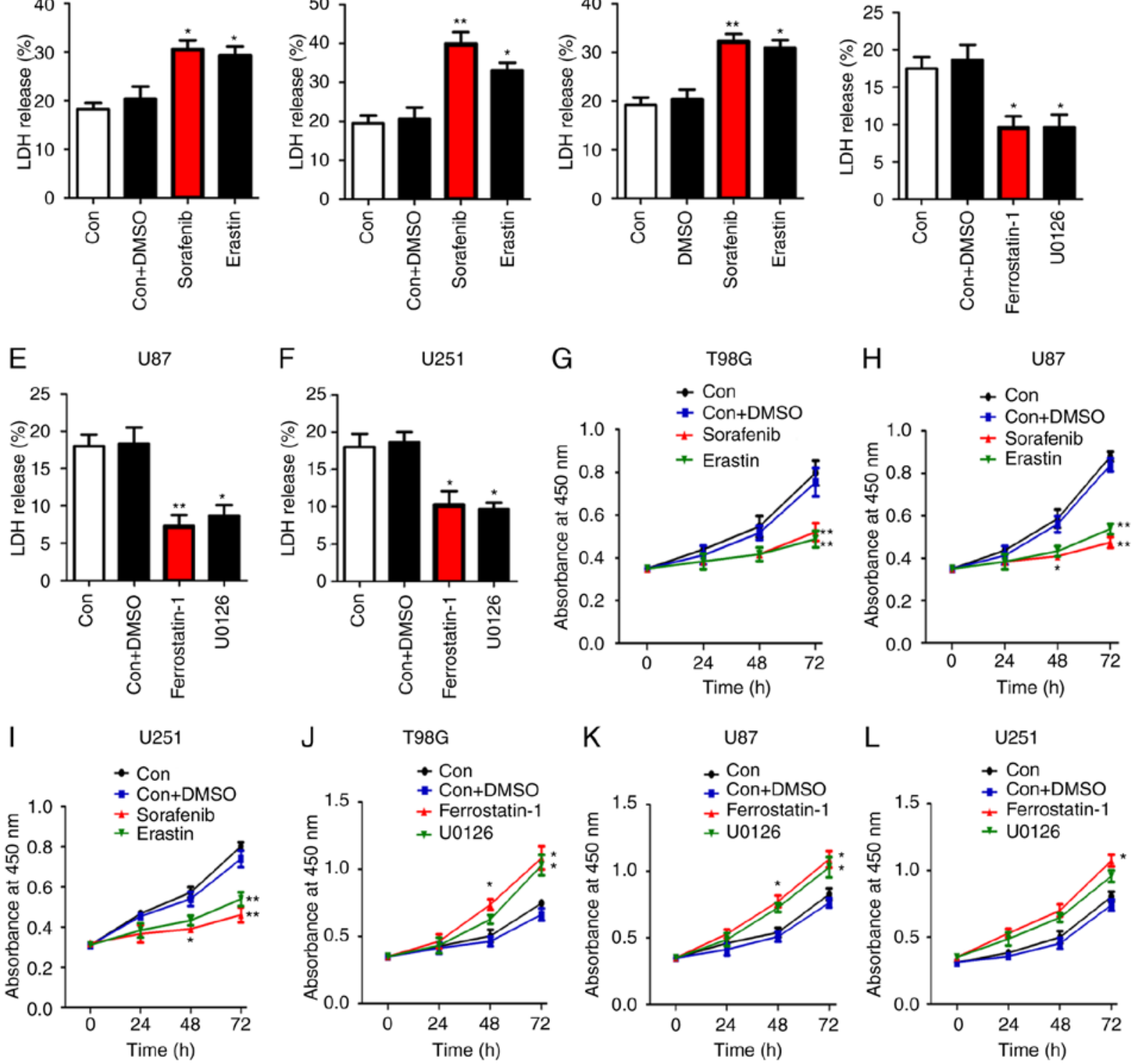

Figure 3. Ferroptosis inhibits proliferation of glioma cells. T98G, U87 and U251 cells were treated with the ferroptosis inducers sorafenib (5 $\mu$ M) or erastin $(10 \mu \mathrm{M})$, or the ferroptosis inhibitors ferrostatin-1 $(1 \mu \mathrm{M})$ or U0126 $(5 \mu \mathrm{M})$, or an equivalent volume of DMSO for 3 days. The cytotoxic substance LDH in (A) T98G, (B) U87 and (C) U251 cells treated with the ferroptosis inducers. The cytotoxic substance LDH in (D) T98G, (E) U87 and (F) U251 cells treated with the ferroptosis inhibitors. Cell viability of (G) T98G, (H) U87 and (I) U251 cells treated with the ferroptosis inducers at different time-points. (J) Cell viability of T98G, (K) U87 and (L) U251 cells treated with the ferroptosis inhibitors at different time-points. $\mathrm{n}=6$ per group. ${ }^{\mathrm{P}} \mathrm{P}<0.05,{ }^{* *} \mathrm{P}<0.01 \mathrm{vs}$. Con $+\mathrm{DMSO}$ $\mathrm{LDH}$, lactate dehydrogenase; con, control.

new form of cell death termed ferroptosis was described as the result of cysteine transfer inhibition and GPx4 inactivation (10). Ferroptosis is a form of cell death dependent on iron and is associated with oxidative damage (34-36). Features of ferroptosis include cytoplasmic and lipidic ROS accumulation, a reduction in mitochondrial volume, an increase in mitochondrial membrane density, rupture or loss of mitochondrial cristae and rupture of the mitochondrial outer membrane (8). Unfortunately, the present study did not obtain typical electron microscope images of ferroptosis. The absence of these data is a limitation of the study and future studies should involve this. Similar to other cell death patterns, ferroptosis is closely associated with certain signaling pathways. Iron accumulation and lipid peroxidation are the key links associated with the occurrence of ferroptosis (37). Ivanov et al (38) demonstrated that the administration of mineral-containing molten iron prior to radiotherapy reduces the efficiency of radiotherapy in a male rat model of glioma, which may be associated with ferroptosis (38). Additional studies on ferroptosis and glioma are limited. Based on the available knowledge, the effects of ferroptosis on gliomas were examined. The protein expression levels of GPx4 in human glioma tissues and different glioma cells were assessed and GPx4 was increased both in vivo and in vitro. Additionally, expression of markers of ferroptosis, including 12-HETE and 15-HETE was reduced. Therefore, it was concluded that ferroptosis is reduced in glioma and it was speculated that ferroptosis reduction may be associated with the pathogenesis of glioma. Notably, there were no female donors at the time of the present study; therefore, the 

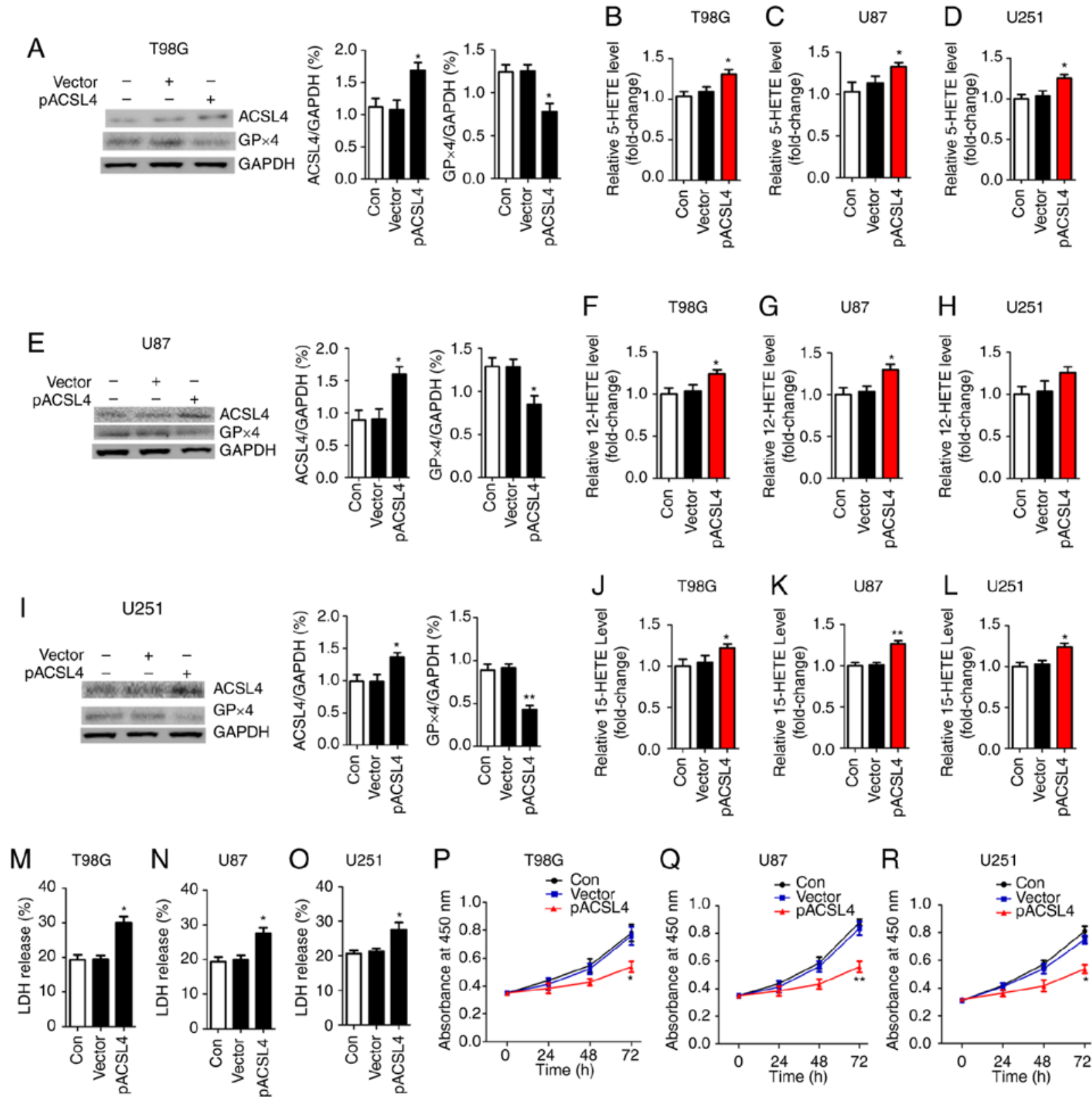

Figure 4. ACSL4 overexpression promotes ferroptosis and inhibits proliferation in glioma cells. Cells were transfected with pcDNA3.1 or pACSL4 for 2 days. (A) Western blots of ACSL4 and GPx4 expression in each group of T98G cells. (B) 5-HETE levels were detected using a 5-HETE kit in each group of (B) T98G, (C) U87 and (D) U251 cells. (E) Western blots of ACSL4 and GPx4 expression in each group of U87 cells. 12-HETE levels were determined using a 12-HETE kit in each group of (F) T98G, (G) U87 and (H) U251 cells. (I) Western blots of ACSL4 and GPx4 expression in each group of U251 cells. 15-HETE levels were assessed using a 15-HETE kit in each group of (J) T98G, (K) U87 and (L) U251 cells. (M) Release of LDH was evaluated using an LDH assay in each group of (M) T98G, (N) U87 and (O) U251 cells. Cell viability of cells in each group of (P) T98G, (Q) U87 and (R) U251 cells at different times. n=6 per group, ${ }^{*} \mathrm{P}<0.05,{ }^{* *} \mathrm{P}<0.01$ vs. Vector. ACSL4, acyl-CoA synthetase long-chain family member 4; HETE, hydroxyeicosatetraenoic acid; GPx4, glutathione peroxidase 4; LDH, lactate dehydrogenase; con, control; pACSL4, pcDNA3.1-ACSL4.

results of the experiments using human glioma tissues may not be convincing for female patients with glioma.

Previous studies have suggested that the progression and poor prognosis of glioma are the result of ferroptosis inhibition. A previous study has indicated that pseudomonic acid B induces ferroptosis in glioma cells by activating Nox 4 and inhibiting xCT (39). Silencing the expression of cystine/glutamate reverse transporter Xc may upregulate ferroptosis and enhance the sensitivity of glioma to the chemotherapeutic drug temozolomide (40). Friedmann Angeli et al (12) reported that Nrf2 overexpression and Keap1-knockdown accelerate cell proliferation and metastasis by inhibiting ferroptosis. ATF4 reduces tumor-mediated neurotoxicity and tumor angiogenesis by increasing ferroptosis (12). Consistent with the aforementioned results, a reduced viability of ferroptosis-induced cells and an increased viability of ferroptosis-inhibited cells were observed. Notably, ferroptosis negatively contributed to proliferation in glioma cells.

At present, three primary molecules have been demonstrated to exert notable regulatory effects on ferroptosis, ACSL4, Xc and GPx4 (30,41-43). Previously, focus has been placed on ACSL4, a vital regulatory molecule for ferroptosis. ACSL4 is a member of the ACSL family and a key enzyme responsible for catalyzing fatty acids with a 12-20 carbon chain length $(44,45)$. 

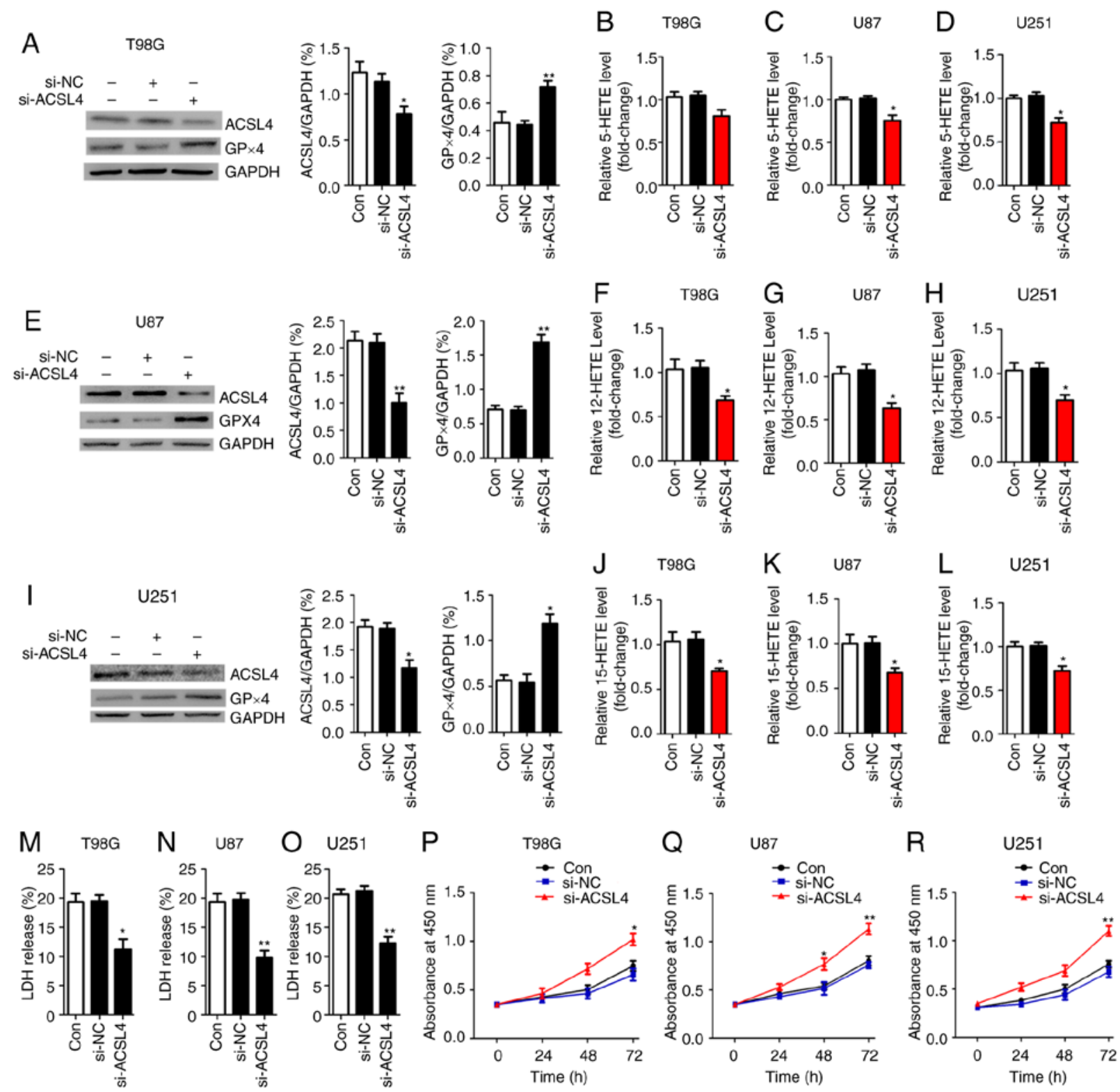

Figure 5. ACSL4 inhibition reduces ferroptosis and induces proliferation in glioma cells. Cells were transfected with si-ACSL4 or a non-specific siRNA as the NC for 2 days. (A) Western blots of ACSL4 and GPx4 expression in each group of T98G cells. (B) 5-HETE levels were detected using a 5-HETE kit in each group of (B) T98G, (C) U87 and (D) U251 cells. (E) Western blots of ACSL4 and GPx4 expression in each group of U87 cells. 12-HETE levels were determined using a 12-HETE kit in each group of (F) T98G, (G) U87 and (H) U251 cells. (I) Western blots of ACSL4 and GPx4 expression in each group of U251 cells. 15-HETE levels were assessed using a 15-HETE kit in each group of (J) T98G, (K) U87 and (L) U251 cells. (K) 15-HETE levels were assessed using a 15-HETE kit in each group in U87 cell. Release of LDH was evaluated using an LDH assay in each group of (M) T98G, (N) U87 and (O) U251 cells. Cell viability of cells in each group of (P) T98G, (Q) U87 and (R) U251 cells at different time-points. $n=6$ per group. ${ }^{*} \mathrm{P}<0.05$, ${ }^{* *} \mathrm{P}<0.01$ vs. si-NC. ACSL4, acyl-CoA synthetase long-chain family member 4; HETE, hydroxyeicosatetraenoic acid; GPx4, glutathione peroxidase 4; LDH, lactate dehydrogenase; si, small interfering; $\mathrm{NC}$, negative control; con, control.

The preferred substrates of ACSL4 are long-chain polyunsaturated fatty acids, such as AA and eicosapentaenoic acid. ACSL4 catalyzes these fatty acids and synthesizes the corresponding coenzyme A $(46,47)$. Recently, ACSL4 was demonstrated to serve as an essential component of ferroptosis through gene screening of Genome-Wide CRISPR and microarray analysis of anti-ferroptosis cell lines (13). Knockdown of ACSL4 inhibited erastin-induced apoptosis in HepG2 and HL60 cells, and ACSL4 overexpression restored the sensitivity of LNCaP and K562 cells to the ferroptosis inducer Erastin (25). A study has additionally shown that the ACSL4-mediated product 5-HETE contributes to ferroptosis (13). As such, ACSL4 is regarded as a sensitive regulator of ferroptosis. However, to the best of our knowledge, whether ACSL4 influences ferroptosis in glioma remains unknown. In the present study, western blotting and immunofluorescence results showed that ACSL4 expression was decreased in glioma tissues compared with normal human brain tissues. Additionally, the protein expression levels of ACSL4 were reduced in glioma cells, consistent with the in vivo results. Therefore, it was hypothesized that the reduction of ferroptosis in glioma may be attributed to a reduction in ACSL4 expression. To validate the role of ACSL4 in ferroptosis in glioma, an ACSL4 overexpression plasmid or si-ACSL4 were transfected into three different glioma 

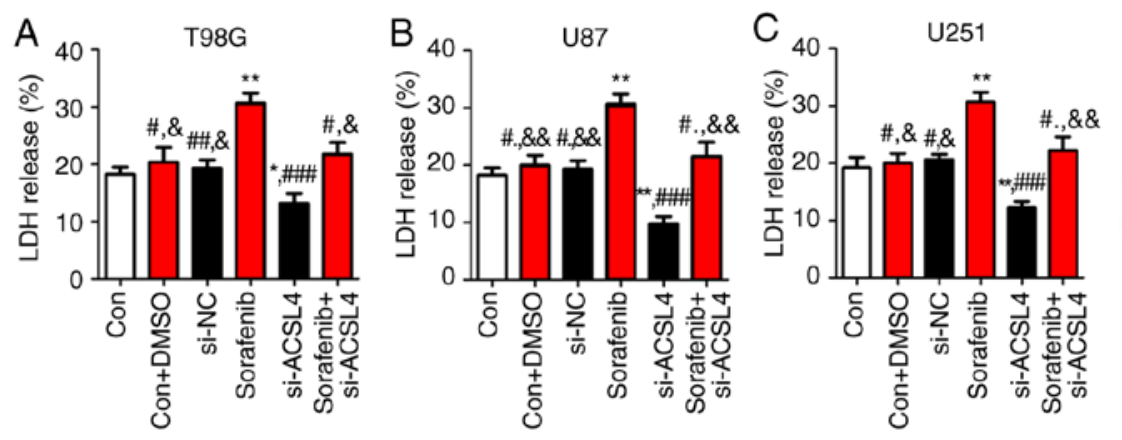

G
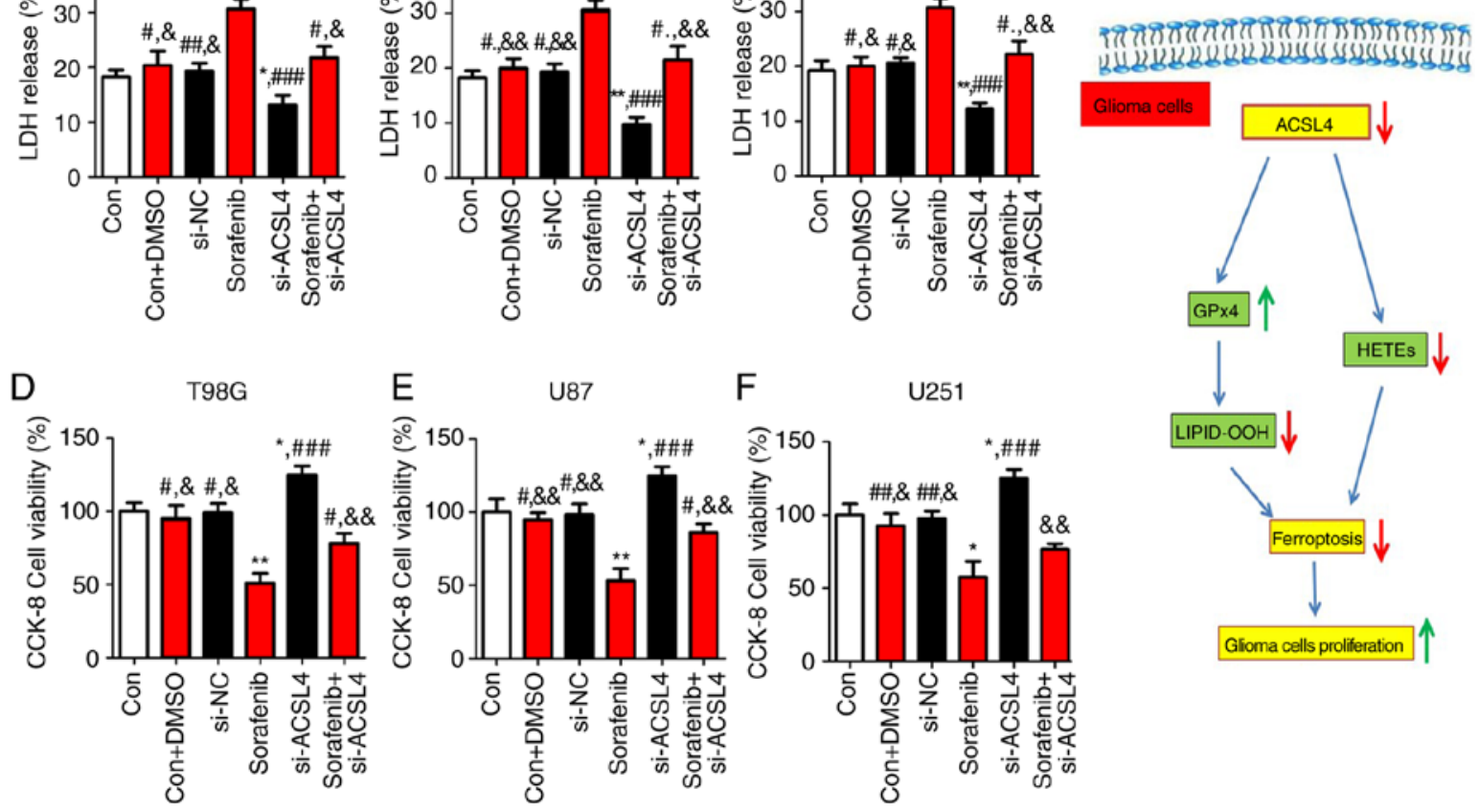

Figure 6. ACSL4 inhibition reduces proliferation by activating ferroptosis in glioma cells. Cells were pre-treated with si-ACSL4 or a non-specific siRNA as the NC, and ferroptosis was induced using sorafenib. (A) LDH levels were detected using a LDH assay in the different groups of (A) T98G, (B) U87 and (C) U251 cells. Cell Counting Kit-8 assays showed the viability of (D) T98G, (E) U87 and (F) U251 glioma cells in the different groups at $72 \mathrm{~h}$ after cells were seeded in 96 -well plates. (G) A schematic summary of the means by which ACSL4 promotes ferroptosis. $n=6$ per group. ${ }^{*} \mathrm{P}<0.05$, ${ }^{* *} \mathrm{P}<0.01$ vs. Con; ${ }^{\#} \mathrm{P}<0.05,{ }^{\# \#} \mathrm{P}<0.01,{ }^{\# \# \#} \mathrm{P}<0.001$ vs. sorafenib, ${ }^{\circledR} \mathrm{P}<0.05$, ${ }^{\& \&} \mathrm{P}<0.01$ vs. si-ACSL4. LIPID-OOH, hydroperoxy-lipid; HETE, hydroxyeicosatetraenoic acid; si, small interfering; ACSL4, acyl-CoA synthetase long-chain family member 4; LDH, lactate dehydrogenase; con, control; NC, negative control.

cell lines. The results suggested that ACSL4 overexpression reduced GPx4 expression, and increased expression of the ferroptosis indicators 5-HETE, 12-HETE and 15-HETE. In addition, ACSL4 expression increased the release of LDH and reduced the survival of glioma cells, confirming that ACSL4 upregulation promoted ferroptosis and decreased proliferation in glioma cells. Conversely, ACSL4-knockdown decreased ferroptosis and promoted proliferation in glioma cells. Taken together, these results suggest that ACSL4 regulates ferroptosis and proliferation in glioma cells, and thus the pathogenesis of glioma.

Thiazolidinediones are selective inhibitors of ACSL4, which have been studied in mouse embryonic fibroblasts (MEFs) and GPx4-deficiency conditional knockout mice. Administration of thiazolidinediones increases the survival rate of MEF cells, and is unable to induce oxidation of membrane lipids and ferroptosis when treated with the ferroptosis inducer RSL3 (13). Additionally, thiazolidinediones prolong the survival time in GPx4-deficient conditional knockout mice treated with a ferroptosis inducer (13). In order to examine the mechanism by which ACSL4 regulates proliferation in glioma cells, additional experiments were performed in the present study. si-ACSL4 alleviated sorafenib-induced LDH release and reduction in cell viability. ACSL4 promoted proliferation of glioma cells by activating ferroptosis. Therefore, ACSL4 expression serves a role in reducing of proliferation of glioma cells by increasing ferroptosis. To show the conclusion more directly, Fig. $6 \mathrm{G}$ presents the proposed mechanism that ACSL4 inhibition directly affects the production of HETEs and directly affects the expression of GPx4, which finally promotes glioma cell proliferation by inhibiting ferroptosis. GPx4 reduces ferroptosis by reducing the production of hydroperoxy-lipid (34).

In summary, the present study demonstrated that ACSL4 suppresses proliferation of glioma cells, at least in part, by activating ferroptosis. The results suggest that ACSL4 may be regarded as a potential therapeutic target for the treatment of glioma. These findings may underlie future studies on pathways involving ACSL4 in a clinical setting.

\section{Acknowledgements}

Not applicable.

\section{Funding}

This study was supported by the National Natural Science Foundation of China (grant. no. 81572489).

\section{Availability of data and materials}

The datasets used and/or analyzed during the current study are available from the corresponding author on reasonable request.

\section{Authors' contributions}

$\mathrm{JC}$ and $\mathrm{YQF}$ wrote the manuscript and participated in the cell experiments. BHL participated in the design of study and cell experiments. HZ collected clinical glioma specimens and analyzed the data. JMW and QXC made substantial 
contributions to the conception and design of the study. All authors read and approved the final manuscript.

\section{Ethics approval and consent to participate}

Procurement of tissues for the present study was approved by the Institutional Ethics Committee of the Faculty of Medicine at Renmin Hospital of Wuhan University (approval no. 2018K-C017), and written informed consent was obtained from each patient.

\section{Patient consent for publication}

Not applicable.

\section{Competing interests}

The authors declare that they have no competing interests.

\section{References}

1. Davis FG and McCarthy BJ: Current epidemiological trends and surveillance issues in brain tumors. Expert Rev Anticancer Ther 1: 395-401, 2001.

2. Norden AD, Drappatz J and Wen PY: Novel anti-angiogenic therapies for malignant gliomas. Lancet Neurol 7: 1152-1160, 2008.

3. Fatehi M, Hunt C, Ma R and Toyota BD: Persistent disparities in survival for patients with glioblastoma. World Neurosurg 120 e511-e516, 2018.

4. Lee A, Youssef I, Osborn VW, Safdieh J, Becker DJ and Schreiber D: The utilization of MGMT promoter methylation testing in United States hospitals for glioblastoma and its impact on prognosis. J Clin Neurosci 51: 85-90, 2018.

5. Alifieris $\mathrm{C}$ and Trafalis DT: Glioblastoma multiforme: Pathogenesis and treatment. Pharmacol Ther 152: 63-82, 2015.

6. Gramatzki D, Frei K, Cathomas G, Moch H, Weller M and Mertz KD: Interleukin-33 in human gliomas: Expression and prognostic significance. Oncol Lett 12: 445-452, 2016.

7. Jiang T, Mao Y, Ma W, Mao Q, You Y, Yang X, Jiang C, Kang C, Li X, Chen L, et al: CGCG clinical practice guidelines for the management of adult diffuse gliomas. Cancer Lett 375: 263-273, 2016.

8. Xie Y, Hou W, Song X, Yu Y, Huang J, Sun X, Kang R and Tang D: Ferroptosis: Process and function. Cell Death Differ 23: 369-379, 2016

9. Latunde-Dada GO: Ferroptosis: Role of lipid peroxidation, iron and ferritinophagy. Biochim Biophys Acta Gen Subj 1861: 1893-1900, 2017.

10. Dixon SJ, Lemberg KM, Lamprecht MR, Skouta R, Zaitsev EM, Gleason CE, Patel DN, Bauer AJ, Cantley AM, Yang WS, et al: Ferroptosis: An iron-dependent form of nonapoptotic cell death. Cell 149: 1060-1072, 2012.

11. Stockwell BR, Friedmann Angeli JP, Bayir H, Bush AI, Conrad M, Dixon SJ, Fulda S, Gascón S, Hatzios SK, Kagan VE, et al Ferroptosis: A regulated cell death nexus linking metabolism, redox biology, and disease. Cell 171: 273-285, 2017.

12. Friedmann Angeli JP, Schneider M, Proneth B, Tyurina YY, Tyurin VA, Hammond VJ, Herbach N, Aichler M, Walch A, Eggenhofer $\mathrm{E}$, et al: Inactivation of the ferroptosis regulator Gpx4 triggers acute renal failure in mice. Nat Cell Biol 16: $1180-1191,2014$

13. Doll S, Proneth B, Tyurina YY, Panzilius E, Kobayashi S, Ingold I, Irmler M, Beckers J, Aichler M, Walch A, et al: ACSL4 dictates ferroptosis sensitivity by shaping cellular lipid composition. Nat Chem Biol 13: 91-98, 2017.

14. Fan Z, Wirth AK, Chen D, Wruck CJ, Rauh M, Buchfelder M and Savaskan N: Nrf2-Keap1 pathway promotes cell proliferation and diminishes ferroptosis. Oncogenesis 6: e371, 2017.

15. Chen D, Fan Z, Rauh M, Buchfelder M, Eyupoglu IY and Savaskan N: ATF4 promotes angiogenesis and neuronal cell death and confers ferroptosis in a xCT-dependent manner. Oncogene 36: 5593-5608, 2017.
16. Killion EA, Reeves AR, El Azzouny MA, Yan QW, Surujon D, Griffin JD, Bowman TA, Wang C, Matthan NR, Klett EL, et al: A role for long-chain acyl-CoA synthetase-4 (ACSL4) in diet-induced phospholipid remodeling and obesity-associated adipocyte dysfunction. Mol Metab 9: 43-56, 2018.

17. Bhutia YD and Ganapathy V: Glutamine transporters in mammalian cells and their functions in physiology and cancer. Biochim Biophys Acta 1863: 2531-2539, 2016.

18. Yu H, Guo P, Xie X, Wang Y and Chen G: Ferroptosis, a new form of cell death, and its relationships with tumourous diseases. J Cell Mol Med 21: 648-657, 2017.

19. Abeti R, Parkinson MH, Hargreaves IP, Angelova PR, Sandi C, Pook MA, Giunti P and Abramov AY: 'Mitochondrial energy imbalance and lipid peroxidation cause cell death in Friedreich's ataxia'. Cell Death Dis 7: e2237, 2016.

20. Wang H, An P, Xie E, Wu Q, Fang X, Gao H, Zhang Z, Li Y, Wang X, Zhang J, et al: Characterization of ferroptosis in murine models of hemochromatosis. Hepatology 66: 449-465, 2017.

21. Doll S and Conrad M: Iron and ferroptosis: A still ill-defined liaison. IUBMB Life 69: 423-434, 2017.

22. Cao JY and Dixon SJ: Mechanisms of ferroptosis. Cell Mol Life Sci 73: 2195-2209, 2016.

23. Kagan VE, Mao G, Qu F, Angeli JP, Doll S, Croix CS, Dar HH, Liu B, Tyurin VA, Ritov VB, et al: Oxidized arachidonic and adrenic PEs navigate cells to ferroptosis. Nat Chem Biol 13: 81-90, 2017

24. Liao J, Tao X, Ding Q, Liu J, Yang X, Yuan FE, Yang JA, Liu B, Xiang GA and Chen Q: SSRP1 silencing inhibits the proliferation and malignancy of human glioma cells via the MAPK signaling pathway. Oncol Rep 38: 2667-2676, 2017.

25. Yuan H, Li X, Zhang X, Kang R and Tang D: Identification of ACSL4 as a biomarker and contributor of ferroptosis. Biochem Biophys Res Commun 478: 1338-1343, 2016.

26. Kumar P, Nagarajan A and Uchil PD: Analysis of cell viability by the lactate dehydrogenase assay. Cold Spring Harb Protoc 2018: 2018.

27. Shan Y, Liu B, Li L, Chang N, Li L, Wang H, Wang D, Feng $\mathrm{H}$, Cheung $\mathrm{C}$, Liao $\mathrm{M}$, et al: Regulation of PINK1 by NR2B-containing NMDA receptors in ischemic neuronal injury. J Neurochem 111: 1149-1160, 2009.

28. Curtis MJ, Bond RA, Spina D, Ahluwalia A, Alexander SP, Giembycz MA, Gilchrist A, Hoyer D, Insel PA, Izzo AA, et al: Experimental design and analysis and their reporting: New guidance for publication in BJP. Br J Pharmacol 172: 3461-3471, 2015.

29. Imai H, Matsuoka M, Kumagai T, Sakamoto $T$ and Koumura T: Lipid peroxidation-dependent cell death regulated by GPx4 and ferroptosis. Curr Top Microbiol Immunol 403: 143-170, 2017.

30. Prabst K, Engelhardt H, Ringgeler S and Hübner H: Basic colorimetric proliferation assays: MTT, WST, and resazurin. Methods Mol Biol 1601: 1-17, 2017.

31. Louandre C, Marcq I, Bouhlal H, Lachaier E, Godin C, Saidak Z, François C, Chatelain D, Debuysscher V, Barbare JC, et al: The retinoblastoma $(\mathrm{Rb})$ protein regulates ferroptosis induced by sorafenib in human hepatocellular carcinoma cells. Cancer Lett 356: 971-977, 2015

32. Geng N, Shi BJ, Li SL, Zhong ZY, Li YC, Xua WL, Zhou H and Cai JH: Knockdown of ferroportin accelerates erastin-induced ferroptosis in neuroblastoma cells. Eur Rev Med Pharmacol Sci 22: 3826-3836, 2018.

33. Li Y, Feng D, Wang Z, Zhao Y, Sun R, Tian D, Liu D, Zhang F, Ning S, Yao J and Tian X: Ischemia-induced ACSL4 activation contributes to ferroptosis-mediated tissue injury in intestinal ischemia/reperfusion. Cell Death Differ 26: 2284-2299, 2019.

34. Forcina GC and Dixon SJ: GPX4 at the crossroads of lipid homeostasis and ferroptosis. Proteomics 19: e1800311, 2019.

35. Zhou B, Liu J, Kang R, Klionsky DJ, Kroemer G and Tang D: Ferroptosis is a type of autophagy-dependent cell death. Semin Cancer Biol: Mar 14, 2019 (Epub ahead of print). doi: 10.1016/j. semcancer.2019.03.002

36. Lei P, Bai T and Sun Y: Mechanisms of ferroptosis and relations with regulated cell death: A review. Front Physiol 10: 139, 2019.

37. Dixon SJ and Stockwell BR: The role of iron and reactive oxygen species in cell death. Nat Chem Biol 10: 9-17, 2014.

38. Ivanov SD, Semenov AL, Mikhelson VM, Kovan'ko EG and Iamshanov VA: Effects of iron ion additional introduction in radiation therapy of tumor-bearing animals. Radiats Biol Radioecol 53: 296-303, 2013 (In Russian). 
39. Wang Z, Ding Y, Wang X, Lu S, Wang C, He C, Wang L, Piao M, Chi G, Luo Y and Ge P: Pseudolaric acid B triggers ferroptosis in glioma cells via activation of Nox4 and inhibition of $\mathrm{xCT}$. Cancer Lett 428: 21-33, 2018.

40. Sehm T, Rauh M, Wiendieck K, Buchfelder M, Eyüpoglu IY and Savaskan NE: Temozolomide toxicity operates in a xCT/SLC7a11 dependent manner and is fostered by ferroptosis. Oncotarget 7 : 74630-74647, 2016.

41. Dixon SJ, Patel DN, Welsch M, Skouta R, Lee ED, Hayano M, Thomas AG, Gleason CE, Tatonetti NP, Slusher BS and Stockwell BR: Pharmacological inhibition of cystine-glutamate exchange induces endoplasmic reticulum stress and ferroptosis. Elife 3: e02523, 2014

42. Wang L, Liu Y, Du T, Yang H, Lei L, Guo M, Ding HF, Zhang J, Wang $\mathrm{H}$, Chen $\mathrm{X}$ and Yan C: ATF3 promotes erastin-induced ferroptosis by suppressing system Xc. Cell Death Differ: Jul 4, 2019 (Epub ahead of print). doi: 10.1038/s41418-019-0380-Z.

43. Xiao FJ, Zhang D, Wu Y, Jia QH, Zhang L, Li YX, Yang YF, Wang H, Wu CT and Wang LS: miRNA-17-92 protects endothelial cells from erastin-induced ferroptosis through targeting the A20-ACSL4 axis. Biochem Biophys Res Commun 515: 448-454, 2019.
44. Soupene E and Kuypers FA: Mammalian long-chain acyl-CoA synthetases. Exp Biol Med (Maywood) 233: 507-521, 2008.

45. Ellis JM, Frahm JL, Li LO and Coleman RA: Acyl-coenzyme A synthetases in metabolic control. Curr Opin Lipidol 21: 212-217, 2010.

46. Coleman RA, Lewin TM, Van Horn CG and Gonzalez-Baró MR: Do long-chain acyl-CoA synthetases regulate fatty acid entry into synthetic versus degradative pathways? J Nutr 132: 2123-2126, 2002.

47. Kang MJ, Fujino T, Sasano H, Minekura H, Yabuki N, Nagura H, Iijima $\mathrm{H}$ and Yamamoto TT: A novel arachidonate-preferring acyl-CoA synthetase is present in steroidogenic cells of the rat adrenal, ovary, and testis. Proc Natl Acad Sci USA 94: 2880-2904, 1997.

(i) $($ This work is licensed under a Creative Commons Attribution-NonCommercial-NoDerivatives 4.0 International (CC BY-NC-ND 4.0) License. 